\title{
Essay
}

\section{Thinking about the Conceptual Foundations of the Biological Sciences}

\author{
M. W. Klymkowsky
}

Molecular, Cellular and Developmental Biology and CU Teach, University of Colorado Boulder, Boulder CO 80309-0347

Submitted April 16, 2010; Revised April 27, 2010; Accepted April 27, 2010

Monitoring Editor: Robert DeHaan

\begin{abstract}
Not until the core concepts of biology are clearly defined and become the focus of instruction and assessment can we expect meaningful improvements in biological literacy and the removal of unnecessary barriers to student engagement.
\end{abstract}

\section{INTRODUCTION}

All too often biology education appears to be defined by trivia-an impression that can alienate students from what is an inherently highly personal and intellectually fascinating subject. How is such a situation possible? I will argue that it stems from common practices that include the following: 1) not seriously considering biology's historical and intellectual foundations; 2) underestimating the need for students to come to biology with a robust grounding in physicochemical principles (thermodynamics, molecular structure and formation, reaction dynamics, and systems thinking); 3) a failure to acknowledge the deeply nonintuitive aspects of biological theory, and 4) a failure to think deeply, or perhaps better put, systematically, about the principles that define biological systems. The typical biology curriculum can, rather accurately I am afraid, be viewed as a fragmented collection of facts and idiosyncratic observations, as opposed to overarching principles that are the equivalent to the laws of physics. This approach leaves students in a conceptual vacuum, trying to memorize facts such as the stages of meiosis, the steps in the Krebs cycle, the components of the electron transport chain, the various lipids in membranes, the components of cellular adhesion junctions, and the posttranslational modifications of histones, etc. Spurred by a recent meeting on Conceptual Assess-

DOI: $10.1187 /$ cbe.10-04-0061

Address correspondence to: M. W. Klymkowsky (michael. klymkowsky@colorado.edu).

(c) 2010 M. W. Klymkowsky. CBE—Life Sciences Education (C) 2010 The American Society for Cell Biology. This article is distributed by The American Society for Cell Biology under license from the author(s). It is available to the public under an Attribution-Noncommercial-Share Alike 3.0 Unported Creative Commons License (http://creativecommons.org/licenses/by-nc-sa/3.0). ment, hosted by the American Society for Biochemistry and Molecular Biology (ASBMB), I attempt to define the core conceptual foundations of modern biology, with the goals of instigating discussion among practitioners and hopefully influencing the "standards and test makers" who all too often embrace an overly ambitious yet incoherent and unteachable model. After some decades of teaching and research, including research into, and reflection upon, student thinking (Klymkowsky, 2007), it appears that there are three pillars upon which all of the biological sciences are based; I refer to these as evolutionary thinking, molecular foundations, and network behavior. Something of a similar exercise (but focused on research rather than teaching) has been carried out by the National Academy of Sciences; its primary emphasis was on the modular and network organization of living systems (National Research Council, 2008).

\section{EVOLUTIONARY THINKING}

Honored more in being trivialized than built into the heart of biology curricula, an evolutionary perspective is essential to understanding biological systems (Dobzhansky, 1973). At the cellular and molecular level, we identify two pillars upon which evolutionary theory rests: the cell theory and an understanding of how information is captured through mutational variation and selection. The cell theory implies continuous descent; biological processes do not begin de novo but rather unfold within this living context. Cellular adaptation and differentiation provide a loose model for one type of unfolding (evolutionary) change, while cellular interactions underlie the behavior of organisms, organ systems, and ecological communities. Biological information theory is based on the recognition that molecular level "noise" (i.e. mutations in the genetic material, DNA, and genetic drift) 


\section{Evolutionary thinking}

- continuity [cell theory]

- stochastic processes [drift \& mutation]

- selection $\rightarrow$ information generation

\section{Molecular foundations}

- thermodynamics: enthalpic \& entropic factors - self-assembly \& systems thinking

- bond formation \& catalysis

- affinity, specificity, and regulation (allostery)

- the molecular level effects of mutation

\section{Network behavior}

- metabolic (non-equilibrium) networks

- adaptive, homeostatic, \& evolving networks

- e.g., molecular, embryological, neural, immune, ecological

provide the raw variation from which natural selection, as proposed by Darwin and Wallace, creates information. It is important to recognize that Darwin and Wallace formed their evolutionary theory without an understanding of how variations arise, and that this ignorance posed a major hurdle to its acceptance (Bowler, 1992). Over the last $\approx 100$ years, studies in genetics and molecular biology have resolved this issue (Jablonka and Lamb, 2005). At the same time, a growing recognition of the high levels of genomic dynamics, including gene duplication and the role of nonadaptive variation (Zhang et al., 2009; Lynch, 2007) suggests that there is a deep and constantly refilling reservoir of genetic variation. Moreover, the universe of genetic variations can be characterized with respect to their phenotypic effects using the coherent system of morphs (i.e., amorphic, hypomorphic, hypermorphic, antimorphic, neomorphic, and neutral [or normomorphic, for mutations with little overt phenotypic effects]) described by Muller (1932).

\section{MOLECULAR FOUNDATIONS}

It is possible to understand genetics from a completely abstract and logical perspective, that is, without knowing how allelic variation arises, how information is encoded, or how such variation modifies or generates traits. However, without a molecular level understanding biological systems are quite mysterious. What is central to such a molecular level understanding? It certainly includes how atoms combine into molecules and how molecules behave, which implies an understanding of the factors that influence their shape, stability, interactions with other molecules, and reactivity. Such an understanding is based on physicochemical principles. Unfortunately, most students have not mastered these ideas before they take biology. The cell, as the basic living unit: 1) is a nonequilibrium system based on coupled chemical reactions; 2 ) its structure and the structures of the macromolecules from which it is composed are determined, to a first level approximation, by spontaneous entropic effects arising from interactions with water; and 3) aside from the electric fields and ion gradients associated with certain membranes, such as those in mitochondria, muscle, and neurons, all interactions within cells are driven by molecular level interactions and entropic (hydrophobic hiding) effects. Moreover, molecular stability, the interactions between molecules, and molecular movement within the cell are driven or influenced by the random thermal jostling of molecules. An understanding of thermal motion as the source of energy driving chemical reactions, together with the importance of reaction coupling, would seem to require, at a minimum, a basic (that is, nonmathematical) understanding of energy transfer, molecular movement/Maxwell-Boltzman distributions, and Le Chatelier's principle.

That order can arise spontaneously at the molecular level is a critical revelation. Understanding such processes, though difficult because they are counterintuitive, would (I suspect) do much to disarm a broad range of creationist disinformation and dispel students' inherent (and justified) confusion. At the same time, it is clear that such molecular level insights are not likely to be delivered efficiently solely within the context of the biology classroom; they depend upon a robust and facile understanding of physicochemical principles. Yet, all too often, the physics taught to students ignores the molecular level behaviors that are relevant to biological systems, and by the same token chemistry instruction often fails to bring students to the level of conceptual confidence needed to understand the molecular behaviors relevant to biological systems. While chemists may blame biologists for the idea that breaking the terminal phosphodiester bond in adenosine triphosphate releases energy, it is clear that students often leave their chemistry courses without understanding that bond breaking always requires energy, while energy is released upon bond formation (M. M. Cooper et al., in preparation). The solution appears to involve an approach based on systems thinking, that is, an explicit consideration of all the bonds broken and formed during a reaction or reaction system. A similar systems perspective is required to fully grasp the logic and inevitability of entropy-driven processes.

\section{NETWORK BEHAVIOR}

Thinking about systems leads naturally to an appreciation that all biological systems are dependent upon network behavior. To understand network behavior, we must explicitly identify the network's components, their properties, interactions, and dynamics, and consider analytically the effects of perturbations, that is, whether the network is stable, adaptive, or evolving. Evolving networks characterize embryonic development, immunological responses, neuronal activity (thinking and memory), disease origin, progression and cure, and ecological stasis and succession. A student's ability to design a molecular system that behaves like the bacterial lac operon or, at the cellular level, determines whether a cell differentiates, divides, or dies, is probably more useful in consolidating learning than is the memorization of gene and protein names. In short, students must go beyond "talking the talk"; they must be able to "walk the walk." Such skills require an accurate appreciation of molecular level function and dynamics and involve analysis and modeling, which will require "making room" in the curriculum for students to master these skills (Klymkowsky, 2009). Such an approach would also enable students to experience for themselves how biological systems work and how their complexity is built from understandable elements. In that light, we find that senior level molecular biology students have serious difficulties generating analytical representations of concepts they might well be assumed to know (C. Trujillo and Klymkowsky, in preparation). 
In sum, I suggest that the key to generating a more effective biological curriculum is to suppress the tendency to be generously inclusive (which often results in a preoccupation with trivia) and to concentrate on foundational knowledge and useful skills. These foundational concepts and skills are, in fact, difficult and require serious immersion and practice to master. Holding students to higher performance (rather than memorization) standards, that is, by assessing their ability to carry out real tasks, is critical to making science real, rather than superficial and needlessly obtuse, for the majority of students. Whether this increases the number of students pursuing a career in biology is not as important as is the demystification of science, particularly given current problems in fully employing the scientists we already produce (Benderly, 2009) and the widespread confusion amid the public of how science works and what it implies in terms of public policy and personal decisions.

\section{ACKNOWLEDGMENTS}

I acknowledge the value of many thoughtful discussions with Melanie Cooper over the last few years on the conceptual foundations of chemistry and biology and how they might be taught more effectively. I also thank the organizers of, and participants in, the ASBMB/National Science Foundation (NSF)-sponsored Conceptual Assessment in Biochemistry and Molecular Biology meeting (March 2010), particularly Ellis Bell, Duane Sears, and Robin Wright, for their inspiration, as well as the University of Colorado Boulder/CU Teach Discipline-based Education Research (DBER) group for providing an ongoing forum for the discussion of a wide range of science education issues. My work in biology education has been supported by NSF grants DUE 0405007 and DUE 0816692 and can be accessed through the beSocratic.colorado.edu and Bioliteracy.colorado.edu websites.

\section{REFERENCES}

Benderly, B. L. (2009). Danger in school labs. Sci. Amer. 303, 18, 20 Bowler, P. J. (1992). The Eclipse of Darwinism: Anti-Darwinian Evolution Theories in the Decades around 1900, Baltimore, MD: Johns Hopkins University Press.

Dobzhansky, T. (1973). Nothing in biology makes sense except in the light of evolution. Amer. Bio. Teach. 35, 125-129.

Jablonka, E., and Lamb, M. J. (2005). Evolution in Four Dimensions: Genetic, Epigenetic, Behavioral, and Symbolic Variation in the History of Life, Cambridge, MA: MIT Press.

Klymkowsky, M. W. (2007). Teaching without a textbook: strategies to focus learning on fundamental concepts and scientific process. CBE Life. Sci. Educ. 6, 190-193.

Klymkowsky, M. W. (2009). Make room for computing. Science 326, 227.

Lynch, M. (2007). The frailty of adaptive hypotheses for the origins of organismal complexity. Proc. Natl. Acad. Sci. USA 104, 85978606.

Muller, H. J. (1932). Sixth Int. Cong. Genet. 1, 213-255.

National Research Council (2008). The Role of Theory in Advancing 21st Century Biology: Catalyzing Transformative Research, Washington, DC: National Academies Press.

Zhang, F., Gu, W., Hurles, M. E., and Lupski, J. R. (2009). Copy number variation in human health, disease, and evolution. Annu. Rev. Genomics. Hum. Genet. 10, 451-481. 